\title{
Evolution morphodynamique d'une plage du littoral Aquitain ; la plage du Truc Vert en Gironde, France
}

\section{Sylvain CAPO ${ }^{1}$, Stéphane BUJAN ${ }^{1}$, Jean-Paul PARISOT ${ }^{1}$, Nadia SENECHAL ${ }^{1}$, Thomas GORIOU ${ }^{2}$}

\author{
${ }^{1}$ Equipe METHYS. UMR 5805-EPOC/OASU, Université Bordeaux 1. Avenue des \\ Facultés, 33405, Talence, France \\ ${ }^{2}$ Centre Littoral de Géophysique .Université La Rochelle, avenue Michel Crépeau \\ 17000 La Rochelle, France
}

\author{
s.capo@epoc.u-bordeaux1.fr \\ s.bujan@epoc.u-bordeaux1.fr \\ paparisot@epoc.u-bordeaux1.fr \\ n.senechal@epoc.u-bordeaux1.fr
}

\section{Résumé :}

L'analyse des MNT de la plage du Truc Vert entre 2003 et 2008, établis sur des levés mensuels lors des marées de vives-eaux, montre une forte variabilité morphologique avec la présence des 4 types caractéristiques de plage intermédiaire avec des fréquences relatives indiquant une dominance de type Transverse Bar and Rip (TBR).

Les morphologies moyennées et volumes de la plage du Truc Vert ont été calculés en terme de saisons et d'années. Cette analyse montre une très bonne corrélation avec l'énergie arrivant à la côte avec une relation inverse : plus l'énergie est importante et plus le volume de la plage est faible. Ce signal reste en corrélation pour les 5 ans d'observations, et met en évidence les fonctionnements saisonniers et annuels tout en soulignant la relative stabilité de cette plage à plus long terme.

\begin{abstract}
:
A long term survey of the truc vert beach, an intermediate double barred beach of the Gironde Atlantic coast, has been realised thanks to topographical GPS survey on a spring tide basis. The methodology applied has been implemented through ATV survey in order to obtain centimetric precision over the three dimensions.

The morphological patterns of the beach have then been analysed face to the wave energy input for a 5 year period since 2003 to 2008 . The studied area, 350m long-shore by 250 to $300 \mathrm{~m}$ cross-shore, is about the "barre-baïne" wavelength that allows the authors to describe the sediment volumetric changes face to wave forcing.
\end{abstract}

DOI: 10.5150/jngcgc.2008.004-C $\quad$ (disponible en ligne - http://www.paralia.fr - available online) 
Despite an important volumetric and morphodynamical seasonal and interannual variability, the authors underline the relative long term stability of the truc vert beach.

\section{Mots-clés :}

Plage intermédiaire à double barre - Morphodynamique - forçage par la houle - GPS survey

\section{$1 \quad$ Introduction}

Le littoral représente un enjeu primordial pour la préservation du milieu face aux changements climatiques en raison de sa situation spécifique à l'interface entre domaines océaniques et continentaux. Les menaces naturelles sont nombreuses et susceptibles d'accroître leur impact destructeur par les effets du réchauffement global (élévation du niveau moyen des mers, augmentation de fréquence des tempêtes...).

Les systèmes côtiers sont extrêmement sensibles aux variations de forçage. La côte Aquitaine présente principalement des systèmes de plages sableuses dominées par la houle dans un environnement mésotidal, parmi les plus difficiles à modéliser (Castelle et al, 2007). De récents travaux ont permis d'établir d'une part un modèle conceptuel de morphologie des plages (Castelle et al, 2007) ainsi que le climat de houle (Butel et al, 2002 ; Abadie et al, 2006).

Afin d'établir les impacts morphologiques des forçages, naturels et/ou anthropiques, un suivi long terme de la plage du Truc vert, au Cap ferret en Gironde, a été mis en place par l'équipe METHYS de l'UMR EPOC depuis 1997. Cette plage est une plage à double barre, ou encore plage à barre et baïne. Ces systèmes de barres sableuses représentent un intérêt croissant car ils agissent comme une protection naturelle contre l'érosion marine et dunaire de la zone littorale (Masselink et al, 2006).

L'analyse morphodynamique présentée ici s'appuie sur un important jeu de données de GPS centimétrique permettant l'obtention de MNT en trois dimensions de la plage intertidale. L'objectif de ce travail est de comprendre le fonctionnement saisonnier et pluri-annuel du système de la plage intertidale en relation avec les données de forçage de la houle.

\section{$2 \quad \underline{\text { Site d'étude }}$}

La plage du Truc Vert, en Gironde, se situe sur la côte Atlantique présentant un linéaire de 250 kilomètres quasi exclusivement composée de plages sableuses entre les estuaires de la Gironde et de 1'Adour (Figure 1), bordée par un système complexe de dunes éoliennes (Pedreros, 2000). L'amplitude moyenne de la marée est de 4,5 m en viveseaux et de $2 \mathrm{~m}$ en mortes-eaux. Au large, la vitesse de ces courants n'excède pas 0,25 
m.s. $\mathrm{s}^{-1}$ (Lorin et al., 1979). A la côte, les courants de marée sont très largement négligeables devant ceux induits par la houle (Castelle et Bonneton, 2002).

Les vagues qui atteignent la côte Aquitaine sont principalement générées par les dépressions nord Atlantique (Castelle et al, 2007) et sont par conséquent très variables et saisonnières. Une importante variabilité interannuelle existe également et peut être reliée à l'index NAO (Dupuis et al, 2006).

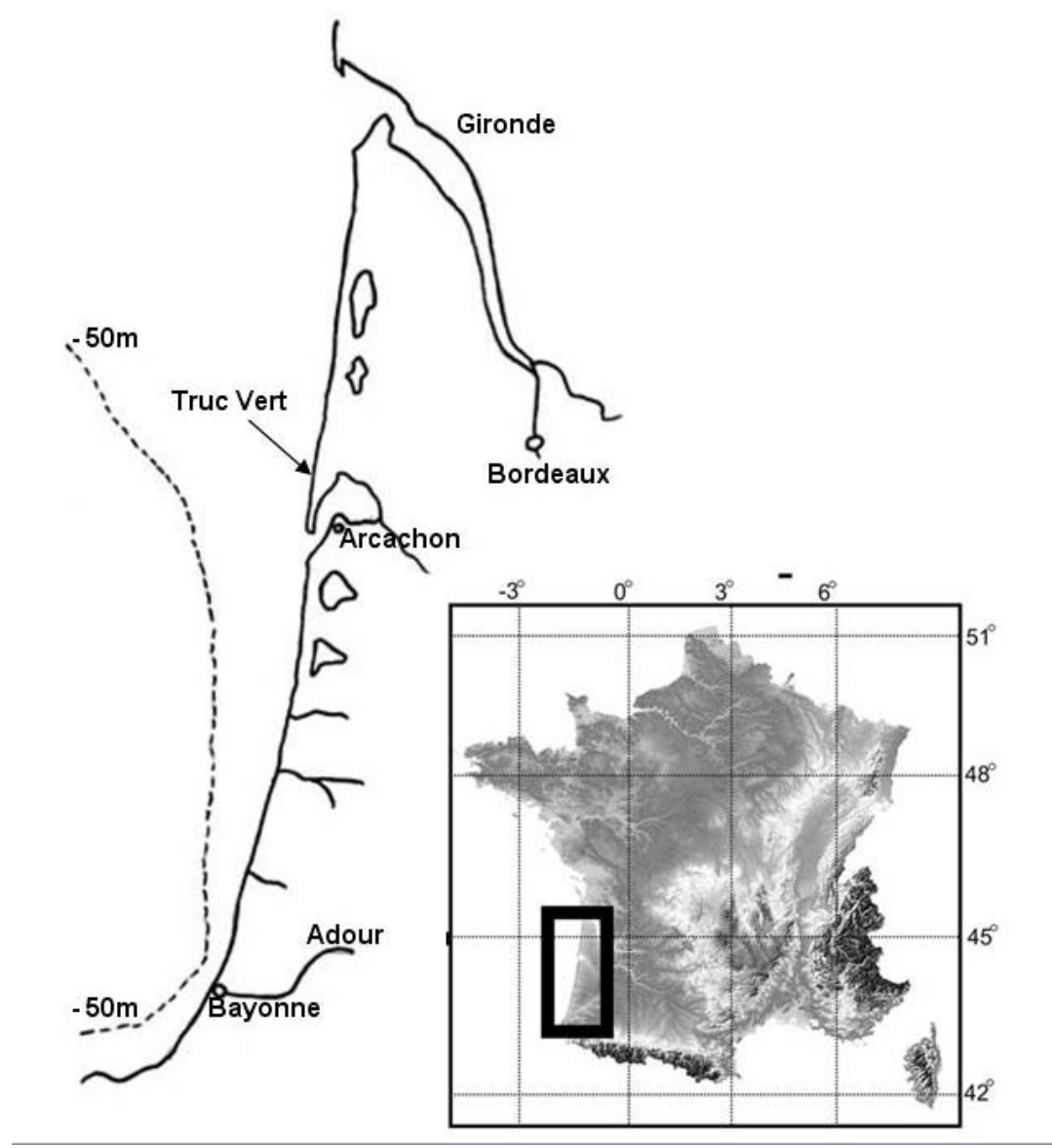

Figure 1 : Localisation du site d'étude sur la côte Aquitaine.

La côte aquitaine est exposée à une forte énergie caractérisée par des houles issues de l'Atlantique Nord principalement d'origine Ouest à Nord Ouest (Butel et al, 2002). Ce climat de vague génère une dérive littorale considérable atteignant $700000 \mathrm{~m}^{3} \mathrm{de}$ sédiment par an dans la région du Truc Vert (Michel et Howa, 1994) alors que ces valeurs sont bien moins considérables (Abadie et al, 2006) vers Capbreton où elles atteignent $40000 \mathrm{~m}^{3}$ par an. Les plages sont majoritairement des plages intermédiaires à double barres selon la classification de Short (1992), Short et Aagaard (1993). La plage du Truc Vert présente généralement une morphologie de type Transverse Bar and Rip (TBR) en ce qui concerne la barre intertidale (Castelle et al, 2007) avec une longueur d'onde moyenne de l'ordre de 400m (Lafon et al, 2002). La plupart du temps, les barres 
sont obliques à la côte et le système de barre externe en croissant présente une morphologie plutôt Rythmic Bar and Beach (RBB) (Froidefond et al., 1990; Lafon et al., 2005) avec une longueur d'onde de l'ordre de $600 \mathrm{~m}$ (Lafon et al., 2004).

\section{$3 \quad$ Méthodologie}

De nombreux paramètres physiques ont été enregistrés depuis 1997 sur la plage du Truc Vert (topographie, trait de côte, profils de plages, mesures de houle) et permettent d'analyser les réponses du système plage. Des données topographiques sous forme de profils de plage, complétées par des relevés au GPS cinématiques depuis 2003, décrivent le développement et l'évolution de la plage sur une portion de $350 \mathrm{~m}$ parallèlement à la côte et sur $250 \mathrm{~m}$ transversalement, du pied de dune au bas de plage. La fréquence d'échantillonnage est basée sur un relevé GPS lors des marées de viveseaux permettant un découvrement maximal de la plage intertidale. Ce site est non anthropisé et reste donc principalement soumis aux forçages naturels de la houle.

La méthodologie a été adaptée pour automatiser la mesure à l'aide d'un Quad couplé à un GPS cinématique. Des méthodes originales ont été développées afin d'aboutir à une cohérence centimétrique dans les trois dimensions sur les jeux de données d'origine très différentes (Parisot 2008, comm.pers). Cette précision permet aux auteurs de présenter des résultats de l'évolution morphodynamique de la plage. La relation entre morphologie et énergie arrivant à la plage est décrite pour la période 2003-2008.

Les forçages de houle utilisés dans ce travail sont issus du modèle WaveWatchIII avec un jeu de données de hauteur significative, direction principale et période pic de la houle toutes les trois heures au nœud $45^{\circ} \mathrm{N}$ et $1.25^{\circ} \mathrm{W}$ de profondeur $55 \mathrm{~m}$, depuis le $1 \mathrm{er}$ janvier 2003 jusqu'au 7 Juin 2007.

\section{$4 \quad$ Résultats}

La plage du Truc vert présente un système plage-dune complet avec les morphologies suivantes ; plage subtidale avec barre en croissant, barre intertidale ou encore système de barre-baïne, une berme en haut de plage intertidale, une dune embryonnaire bien formée, une dune blanche et une dune grise formant la dune littorale (Figure 2). 


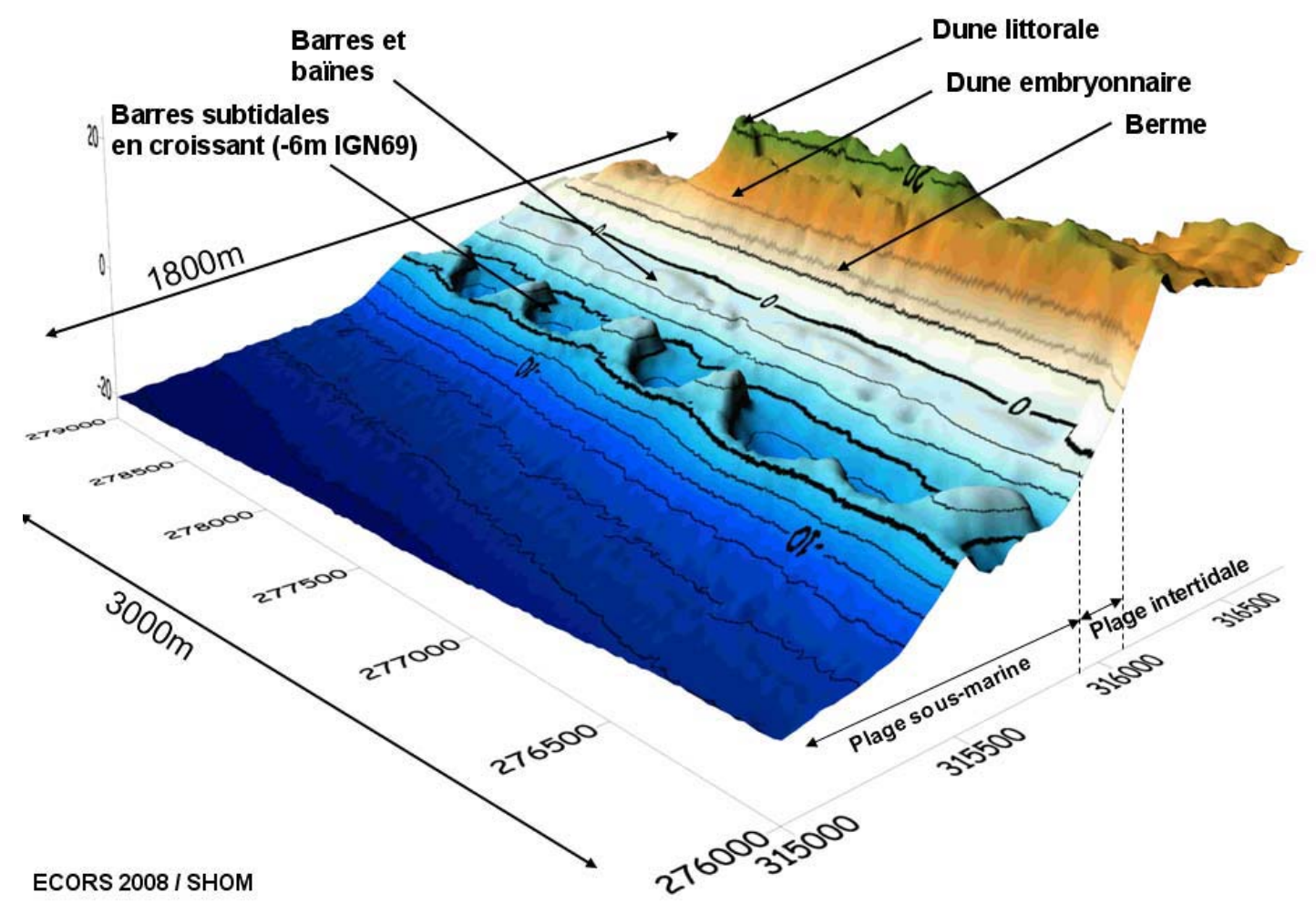

Figure 2 : Profil 3D type de la plage du Truc Vert. Les coordonnées sont en Lambert III Sud. (Données ECORS2008 / SHOM)

Le calcul du volume de la plage du truc vert est effectué sur une même grille commune à tous les relevés sur une portion de plage de $350 \mathrm{~m}$ long-shore sur $250 \mathrm{~m}$ cross-shore. La limite inférieure est de $-3 \mathrm{~m}$ (IGN69) ce qui correspond à une profondeur légèrement inférieure à la limite de plage intertidale. La limite supérieure est définie comme étant la pente de la dune embryonnaire et est constante pour tous les relevés. Ce volume calculé tient donc compte à la fois de la variation de morphologie de la plage intertidale ainsi que de celle du pied de dune. Cet indicateur est ainsi caractéristique des tendances à l'accrétion ou érosives des périodes considérées sans cependant focaliser sur une entité morphologique particulière de la plage. L'énergie moyenne par tranche de trois heures atteint la valeur de $36000 \mathrm{~J}$ par mètre linéaire de plage par seconde, ce qui correspond à une houle de hauteur significative moyenne de $1,5 \mathrm{~m}$, une direction principale de $270^{\circ}$ et une période pic de 9,5 secondes. Les valeurs extrêmes obtenues par le modèle sont respectivement pour les minima et maxima, pour les hauteurs significatives de $0,17 \mathrm{~m}$ et $8,75 \mathrm{~m}$, pour les périodes pic de 2,7 à 19,7 secondes.

La figure 3 montre la relation entre l'évolution temporelle de l'énergie arrivant à la côte et le volume de la plage avec une bonne corrélation entre les épisodes énergétiques et les phases d'érosion ainsi qu'entre les épisodes de faible énergie et les phases d'accrétion. Il apparaît cependant de nombreux épisodes de faible énergie caractérisés par de l'érosion ou inversement des périodes de fortes énergie où l'érosion domine. Il 
faut ainsi observer attentivement la chronologie des évènements et considérer " l' histoire » récente de la plage. La relation entre flux d'énergie à la côte et volume global de plage est ainsi non linéaire avec cependant une tendance générale caractérisée par des évènements de tempête érosifs et des périodes de calme hydrodynamique associés à de l'accrétion, notamment durant l'été.

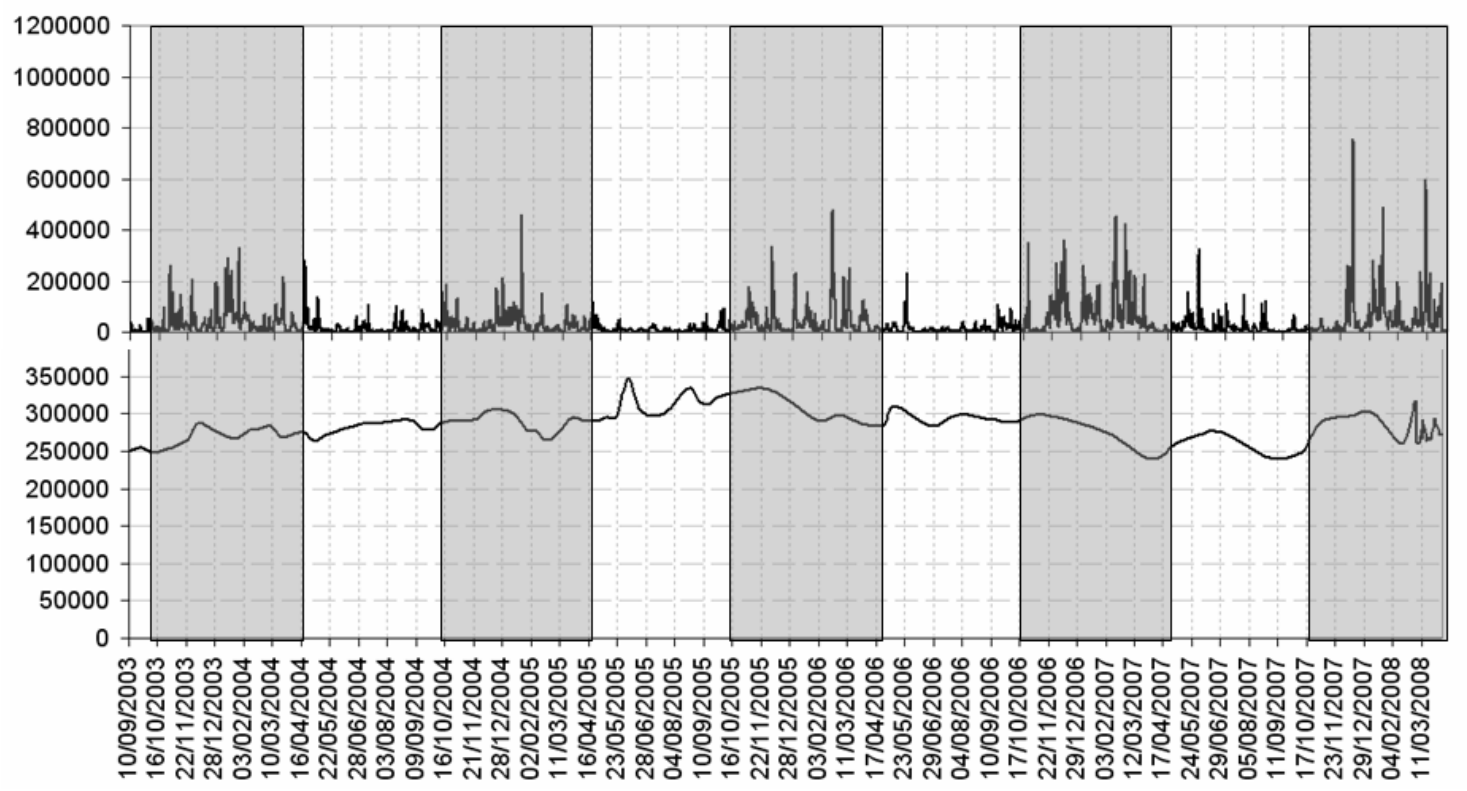

Figure 3 : Evolution temporelle du flux d'énergie instantané (en Joule par mètre linéaire de plage par seconde) en haut et du volume de plage (en mètres cubes) en bas de Septembre 2003 à Avril 2008. Les zones grisées correspondent aux périodes hivernales.

La figure 4 montre la variation cumulée du volume de plage depuis septembre 2003, le volume étant normalisé à 0 pour la première mesure. Une tendance générale d'accrétion est observée de septembre 2003 à fin $2005\left(+80000 \mathrm{~m}^{3}\right)$, puis on observe une tendance générale à l'érosion jusqu'à mi $2007\left(-90000 \mathrm{~m}^{3}\right)$ et enfin à nouveau une tendance à l'accrétion jusqu'en début $2008\left(+50000 \mathrm{~m}^{3}\right)$. La variation moyenne de volume de plage entre deux relevés consécutifs atteint $11300 \mathrm{~m}^{3}$ de sable, correspondant grossièrement à $33 \mathrm{~m} 3$ par mètre linéaire de plage, soit à $1 \mathrm{~m}^{3} \cdot \mathrm{m}^{-1} \cdot \mathrm{j}^{-1}$. De fortes variations sont observées avec une érosion maximale qui atteint $-1400 \mathrm{~m} 3 / \mathrm{j}$ entre les 12 et 28 janvier 2005 soit -4 $\mathrm{m}^{3} / \mathrm{m} / \mathrm{j}$ alors que l'accrétion maximale observée atteint $+2030 \mathrm{~m}^{3} / \mathrm{j}$ entre le 28 avril et 10 mai 2006 soit $+6 \mathrm{~m}^{3} / \mathrm{m} / \mathrm{j}$. 


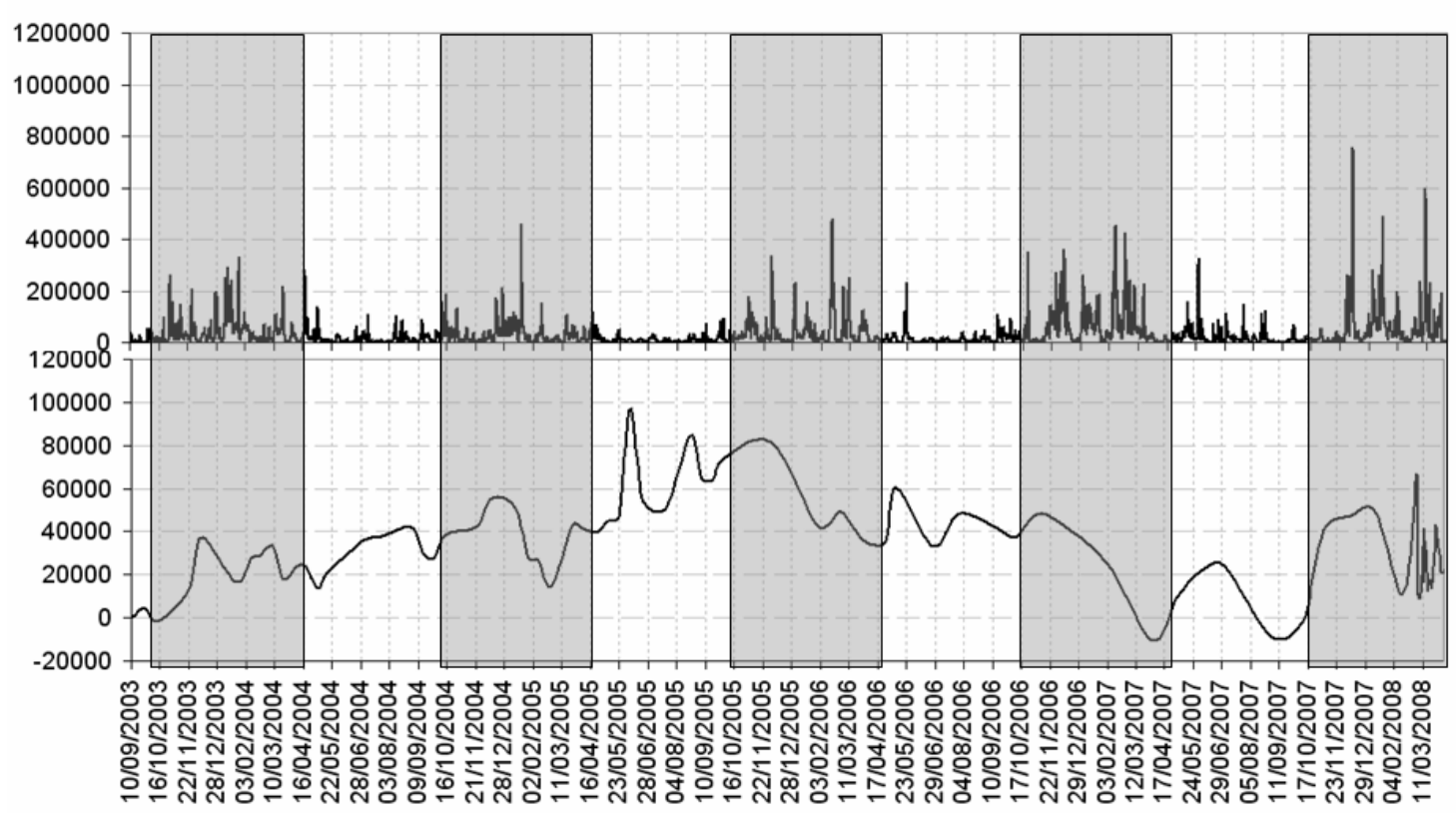

Figure 4 : Evolution temporelle du Flux d'énergie arrivant à la côte (en $\mathrm{J} / \mathrm{m} / \mathrm{s})$ en haut, et Variation cumulée du volume de plage normalisé à 0 pour le premier relevé du 10 septembre 2003, en bas.

Les fonctionnements annuels et saisonniers ont été mis en évidence par le calcul moyen des volumes de plage et de flux d'énergie arrivant à la côte qui montrent une très nette corrélation avec relation inverse entre les deux paramètres (Figure 5).

Le flux d'énergie calculé sur 10 ans entre 1997 et 2007 indique une valeur moyenne de $36000 \mathrm{~J} / \mathrm{m} / \mathrm{s}$ avec un flux maximal pour $2002(42000 \mathrm{~J} / \mathrm{m} / \mathrm{s})$ puis 2000 et $1999(40000$ $\mathrm{J} / \mathrm{m} / \mathrm{s}$ ) et un flux minimal en 2005 avec seulement $24000 \mathrm{~J} / \mathrm{m} / \mathrm{s}$. Il apparait cependant très nettement que le volume de sédiment de la plage du Truc Vert est inversement relié au flux d'énergie, tant en terme de saison que d'interannualité. Les morphologies les plus couramment observées en été, lors des épisodes de faible énergie sur des durées relativement longues sans épisode énergétique sont les Low Tide Terrace (LTT). Lors de la croissance progressive de l'énergie arrivant à la côte durant l'automne, l'hiver voire le printemps, on voit alors se succéder les autres types morphologiques passant du LTT au TBR, qui est le type le plus représenté statistiquement (50 à $60 \%$ d'occurrence) puis aux RBB et LBT lors d'alternance d'épisodes de fortes et de faibles énergie. Les temps de réponses du système plage ne sont pas clairement identifiés dans ce travail, en raison d'une part de la fréquence d'échantillonnage sélectionnée car les relevés sont effectués lors des basses mers de vives-eaux uniquement. 

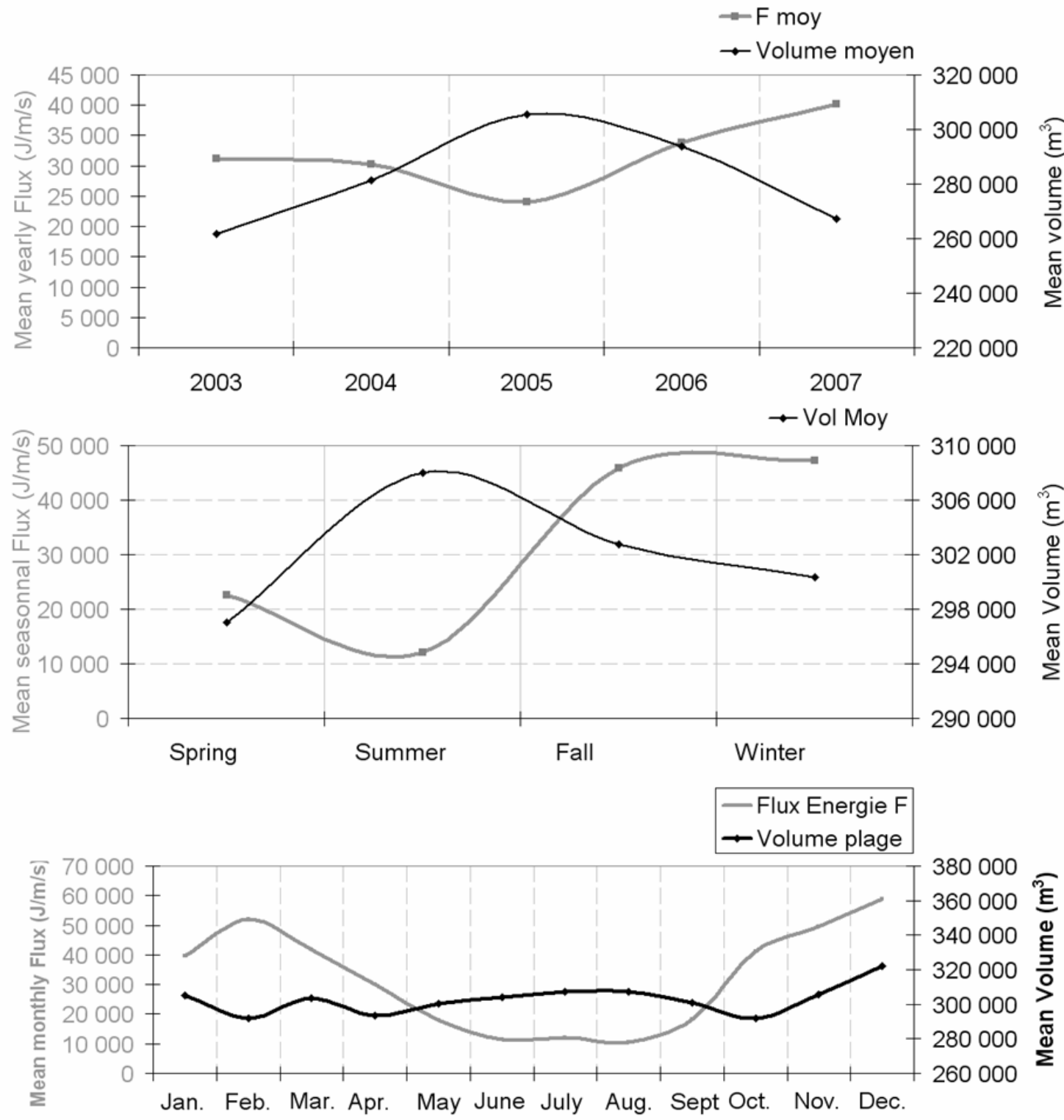

Figure 5 : Evolution annuelle (en haut), saisonnière (milieu) et mensuelle (en bas) du volume de plage en fonction du flux d'énergie arrivant à la côte.

\section{$5 \quad$ Conclusions}

La portion de plage étudiée montre une grande variabilité de morphologie avec la présence des quatre types morphologiques de plage intermédiaire allant du LTT au LBT 
pour une énergie arrivant à la côte croissante. La fréquence relative de chacun de ces états montre une prédominance statistique au TBR (50 à 60\%) suivi par un état LTT (entre 15 et $20 \%$ ) et enfin les deux derniers états RBB et LBT pour des épisodes plus énergétiques entre 10 et $12 \%$ chacun avec cependant la limitation due à la couverture de la zone intertidale exclusivement, ne permettant pas toujours d'être formel sur l'état de la plage. La fréquence d'échantillonnage ne permet pas non plus de mettre en évidence les temps de réponses du système plage pour les passages d'un état à un autre, ce qui fera l'objet d'une étude ultérieure grâce aux données de la campagne ECORS 2008.

L'enregistrement temporel du volume de plage montre néanmoins une relative stabilité de la plage intertidale du truc vert avec une importante variabilité morphologique et volumétrique de la plage sans toutefois marquer de réelle tendance à l'érosion ou à l'accrétion.

\section{$6 \quad$ Références bibliographiques}

1.Abadie, S., Butel, R., Mauriet, S., Morichon, D., Dupuis, H., 2006. Wave climate and longshore drift on the South Aquitaine coast. Continental Shelf Research, vol 26, pp 1924-1939.

2.Butel, R., Dupuis, H., Bonneton, P., 2002. Spatial Variability of Wave Conditions on the French Atlantic Coast using In-Situ Data. J. Coastal Res. SI 36, 96-108.

3.Castelle, B. et Bonneton, P. (2002). Wave-induced currents over the Aquitanian coast sand bars. 8th International Symposium on Oceanography of the Bay of Biscay

4.Castelle, B., Bonneton, P., Dupuis, H., Senechal, N., 2007. Double bar beach dynamics on the high-energy meso-macrotidal French Aquitanian Coast: A review. Marine Geology, vol 245, pp 141-159

5.Froidefond, J.-M., Gallissaires, J.-M., Prud'homme, R., 1990. Spatial variation in sinusoidal on a crescentic nearshore bar: application to the Cap Ferret Coast. J. Coastal Res. 6, 927-942.

6.Lafon, V., Dupuis, H., Howa, H., Froidefond, J.-M., 2002. Determining ridge and runnel longshore migration rate using spot imagery. Oceanologica Acta 25, 149-158.

7.Lafon, V., De Melo Apoluceno, D., Dupuis, H., Michel, D., Howa, H., Froidefond, J.M., 2004. Morphodynamics of nearshore rhythmic sandbars in a mixed-energy environment (Sw France):i. mapping beach changes using visible satellite imagery. Estuar. Coast. Shelf Sci. 61 (2), 289-299.

8.Lafon, V., Dupuis, H., Butel, R., Castelle, B., Michel, D., Howa, H., De Melo Apoluceno, D., 2005. Rhythmic sub-tidal and inter-tidal bar morphology and dynamics in a mixed-energy environment. part II: physical forcing analysis. Estuar. Coast. Shelf Sci. 65 (3), 449-462.

9.Lorin J., Viguier J., Migniot C. (1979). Etude en nature de la côte aquitaine entre la pointe de grave et l'embouchure de l'Adour. L.C.H.F., rapport non publié 10.Masselink G., Kroon A. et Davidson-Arnott R. G. D. (2006). Morphodynamics of intertidal bars in wave dominated coastal settings - A review. Geomorphology 73 (1 - 2) : 33 - 49 
11.Michel, D., Howa, H., 1994. Morphological evolution of a littoral sand bank, modelisation of its dynamics. Annals Geophysical, European Geophysical Union, Part II, 12 , p. 240

12.Pedreros, R., 2000. Quantification et modélisation du transport éolien au niveau des zones côtières-application au littoral aquitain. Ph.D. thesis, University Bordeaux I, in French, unpublished.

13. Short, A.D., 1992. Beach systems of the central netherlands coast: processes, morphology and structural impacts in a storm driven multi-bar system. Mar. Geol. 107, 103-137.

14.Short, A.D., Aagaard, T., 1993. Single and multi-bar beach change models. J. Coast. Res. SI 15, 141-157. 